\title{
Uncertainty Assessment of Lossy and Dispersive Lines in SPICE-Type Environments
}

\author{
Paolo Manfredi, Student Member, IEEE, Dries Vande Ginste, Senior Member, IEEE, \\ Daniël De Zutter, Fellow, IEEE, Flavio G. Canavero, Fellow, IEEE
}

\begin{abstract}
This contribution presents an alternative modeling strategy for the stochastic analysis of high-speed interconnects. The proposed approach takes advantage of the polynomial chaos framework and a fully SPICE-compatible formulation to avoid repeated circuit simulations, therefore alleviating the computational burden associated to traditional sampling-based methods like Monte Carlo. Nonetheless, the technique offers very good accuracy and the opportunity to easily simulate complex interconnect topologies which include lossy and dispersive transmission lines, thus overcoming the limitations of previous publications. Application examples involving the stochastic analysis of onchip and on-board interconnects validate the methodology and conclude the paper.
\end{abstract}

Index Terms-Circuit design, circuit simulation, polynomial chaos, SPICE, stochastic analysis, transmission lines, uncertainty.

\section{INTRODUCTION}

The boost towards very-large-scale integration (VLSI) is stressing the impact of manufacturing tolerances on the performance of electronic circuits and interconnects. The desirability of performing right-the-first-time designs gave raise to a very active research field focused on the statistical assessment of high-speed links [1]. Nevertheless, traditional Monte Carlo analysis is often inefficient due to the slow convergence rate and the lack of an explicit relation between the uncertain response and the random system parameters. Because of this, interconnect designers are seeking for expedite and reliable stochastic modeling strategies to assess variability in the early design phase [2].

In this framework, the so-called polynomial chaos (PC) technique drew much attention in the electrical engineering community. This methodology is based on spectral expansions of random processes in terms of Hermite [3] or other orthogonal polynomials, according to the distribution of the input random variables [4]. PC can be combined with a Galerkin approach, thus becoming a stochastic Galerkin method (SGM). This allows to recast the original stochastic problem in terms of a larger but deterministic one, whose solution provides the unknown expansion coefficients [4].

The PC-SGM approach has been successfully applied to electrical networks described by modified nodal analysis

P. Manfredi and F. Canavero are with the Dipartimento di Elettronica e Telecomunicazioni, EMC Group, Politecnico di Torino, Torino 10129, Italy (e-mail: paolo.manfredi@polito.it; flavio.canavero@polito.it).

D. Vande Ginste and D. De Zutter are with the Department of Information Technology, Electromagnetics Group, Ghent University, Ghent 9000, Belgium (e-mail: dries.vandeginste@ugent.be; daniel.dezutter@ugent.be).
(MNA) equations [5], [6]. However, ad hoc MATLAB implementations or customized software were required for the solution of the resulting augmented MNA equations. For design flexibility, integration into standard circuit analysis software, such as SPICE or Agilent's ADS, was imperative. A SPICE-like code for the stochastic analysis of lumped circuits was presented in [7], but still based on a customized solution engine with specific libraries for the different kinds of stochastic circuit components.

However, all the above applications lacked the inclusion of distributed transmission-line elements, which are key components for modern high-speed designs. Although a stochastic MTL can be in principle analyzed as a cascade of equivalent lumped sections (see, e.g., [5] or [8]), this approach is not suitable to accurately model high-frequency and dispersive phenomena, such as delay or slow-wave and skin effects. Moreover, it requires a large number of additional nodes, thus also reducing the efficiency.

An application of PC-SGM to distributed multiconductor transmission lines (MTLs) was first presented in [9] and then improved in [10], where the combination with parametrized macromodeling made the inclusion of complex frequencydependent wave mechanisms efficient and straightforward. However, the approach was limited to frequency-domain analysis and basic (source-line-load) configurations. The integration of the above methodology in SPICE-like design environments would allow the analysis, either transient or AC, of arbitrary network topologies. A first attempt was proposed in [11], but the discretization scheme commonly used by the PC-SGM partially hindered the implementation, which was limited to lossless and dispersion-free lines. This issue has been solved with a better discretization scheme [12], which opens the door to a SPICE-compatible implementation of lossy and dispersive lines [13].

In this paper, the new scheme is used to provide an efficient strategy for the simulation of generic on-chip and on-board interconnects in a SPICE-type environment, by outlining a systematic approach to extend the work in [13], where only a source-line-load configuration was considered, to the simulation of arbitrary network topologies. The relevance of this approach is exemplified by a number of illustrative applications. The new approach, for the first time, allows a PC-SGMbased statistical (transient) analysis of distributed networks with uncertain parameters, thus overcoming the limitations of previous solutions and providing circuit designers with a powerful and SPICE-compatible tool for a quick statistical assessment of the overall interconnect response. 


\section{Stochastic Simulation of Electrical Networks}

Due to the presence of random circuit components, voltages and currents in the network also become stochastic. According to the PC theory [3], [4], stochastic quantities (in this case, node voltages and branch currents) can be expanded using a proper multivariate polynomial basis $\left\{\phi_{k}\right\}_{k=0}^{P}$, as follows:

$$
V(\boldsymbol{\xi}) \approx \sum_{k=0}^{P} V_{k} \phi_{k}(\boldsymbol{\xi}), \quad I(\boldsymbol{\xi}) \approx \sum_{k=0}^{P} I_{k} \phi_{k}(\boldsymbol{\xi}),
$$

where $\boldsymbol{\xi}=\left[\xi_{1}, \ldots, \xi_{r}\right]$ encompasses all the independent random circuit parameters, and $V_{k}$ and $I_{k}$ are expansion coefficients to be determined. The coefficients $V_{k}$ and $I_{k}$ become then the new unknowns of the problem. The polynomials $\left\{\phi_{k}\right\}$ are traditionally chosen to be orthogonal with respect to the inner product defined as

$$
\langle f, g\rangle=\int_{\Omega} f(\boldsymbol{\xi}) g(\boldsymbol{\xi}) w(\boldsymbol{\xi}) d \boldsymbol{\xi},
$$

with $\Omega$ the support of $\boldsymbol{\xi}$ and $w(\boldsymbol{\xi})$ its probability density function (PDF). We will come back to this choice and further refine it in Section IV.

Expressions (1) apply both in frequency and time domain and provide explicit relations between the stochastic voltages and currents and the random input parameters, thus allowing a fast extraction of statistical information. For instance, the mean and variance are readily obtained as

$$
\mathrm{E}\{V(\boldsymbol{\xi})\}=V_{0}, \quad \mathrm{E}\{I(\boldsymbol{\xi})\}=I_{0}
$$

and

$$
\operatorname{Var}\{V(\boldsymbol{\xi})\}=\sum_{k=1}^{P} V_{k}^{2}\left\|\phi_{k}\right\|^{2}, \quad \operatorname{Var}\{I(\boldsymbol{\xi})\}=\sum_{k=1}^{P} I_{k}^{2}\left\|\phi_{k}\right\|^{2},
$$

respectively, where $\left\|\phi_{k}\right\|^{2}=\left\langle\phi_{k}, \phi_{k}\right\rangle$ is the norm of the polynomials and is analytically known $\forall k$. Higher order moments or distribution functions can be computed by numerically sampling (1), this procedure being fast thanks to the analyticity of the expressions.

In order to avoid repeated circuit simulations of the stochastic electrical network, the strategy for the computation of the PC-expansion coefficients is to obtain, by means of the SGM, new and deterministic constitutive equations, which relate the coefficients and are directly implementable in a SPICE-type environment. It is important to observe that in a circuit with $N$ nodes and $B$ branches, there will be $N(P+1)$ unknown voltage coefficients and $B(P+1)$ unknown current coefficients, with

$$
P+1=\frac{(p+r) !}{p ! r !},
$$

where $r$ is the number of random parameters and $p$ is the expansion order (for practical applications, choosing $p=2$ already provides very good accuracy). This suggests to associate an additional node to each new voltage variable and a branch to each new current variable. The problem is then interpreted as an equivalent, augmented network, where the nodes are connected by appropriate branch elements, defined by the circuit interpretation of the new constitutive equations. The solution then amounts to performing a single circuit simulation of a modified, deterministic network, instead of running a large number of Monte Carlo simulations of the original, stochastic network.

\section{New Constitutive Equations}

The sought-for deterministic relations are readily obtained with the expansion and Galerkin projection of the (stochastic) governing equations of the random circuit elements [11]. For instance, MTLs are described by the so-called telegrapher's equations, which in presence of variations in their parameters can be written in a stochastic fashion as

$$
\begin{aligned}
\frac{d}{d z} \mathbf{V}(z, \omega, \boldsymbol{\xi}) & =-[\mathbf{R}(\omega, \boldsymbol{\xi})+j \omega \mathbf{L}(\omega, \boldsymbol{\xi})] \mathbf{I}(z, \omega, \boldsymbol{\xi}), \\
\frac{d}{d z} \mathbf{I}(z, \omega, \boldsymbol{\xi}) & =-[\mathbf{G}(\omega, \boldsymbol{\xi})+j \omega \mathbf{C}(\omega, \boldsymbol{\xi})] \mathbf{V}(z, \omega, \boldsymbol{\xi}),
\end{aligned}
$$

where $\omega$ is the angular frequency, whilst for a $n$-conductor line $\mathbf{R}, \mathbf{L}, \mathbf{G}, \mathbf{C}$ are the $n \times n$ p.u.l. resistance, inductance, conductance and capacitance matrices, respectively, and $\mathbf{V}$ and $\mathbf{I}$ are $n$-vectors collecting the voltages and currents along the line. The random vector $\boldsymbol{\xi}$ in (6) identifies the quantities affected by random variations.

The randomness of the p.u.1. matrices suggests they can be expanded analogously to (1), i.e.

$$
\mathbf{A}(\omega, \boldsymbol{\xi}) \approx \sum_{k=0}^{P} \mathbf{A}_{k}(\omega) \phi_{k}(\boldsymbol{\xi}),
$$

where A stands for any of the four p.u.l. matrices in (6). The computation of the coefficients $\mathbf{A}_{k}$ is a fundamental step which is addressed in the next section. Substitution of (1) and (7) into (6) and application of Galerkin projection yield the sought-for deterministic relations for the voltage and current coefficients [10], which can be written in matrix form as follows

$$
\begin{aligned}
\frac{d}{d z} \tilde{\mathbf{V}}(z, \omega) & =-[\tilde{\mathbf{R}}(\omega)+j \omega \tilde{\mathbf{L}}(\omega)] \tilde{\mathbf{I}}(z, \omega), \\
\frac{d}{d z} \tilde{\mathbf{I}}(z, \omega) & =-[\tilde{\mathbf{G}}(\omega)+j \omega \tilde{\mathbf{C}}(\omega)] \tilde{\mathbf{V}}(z, \omega),
\end{aligned}
$$

where $\tilde{\mathbf{R}}, \tilde{\mathbf{L}}, \tilde{\mathbf{G}}$ and $\tilde{\mathbf{C}}$ are new $n(P+1) \times n(P+1)$ p.u.l. deterministic (block) matrices, whereas $\tilde{\mathbf{V}}$ and $\tilde{\mathbf{I}}$ are vectors collecting all the $n(P+1)$ unknown PC-coefficients for the voltages and currents in the MTL. The augmented p.u.l. matrices can be easily constructed using the following expression for their $n \times n$ blocks

$$
\tilde{\mathbf{A}}_{i j}=\sum_{k=0}^{P} \mathbf{A}_{k} \alpha_{k j i},
$$

where $\alpha_{k j i}=\left\langle\phi_{k} \phi_{j}, \phi_{i}\right\rangle /\left\langle\phi_{i}, \phi_{i}\right\rangle$ are merely real numbers, which can be computed by means of analytical formulae.

The deterministic relations in (8) can be interpreted as pertinent to a MTL with a larger number of terminals and connecting the $2 n(P+1)$ nodes which arise from the expansion of the original $2 n$ stochastic node voltages, as illustrated in Fig. 1 for the case $n=2$ and $P=2$. Hence, the original stochastic transmission line can be replaced, in the augmented 

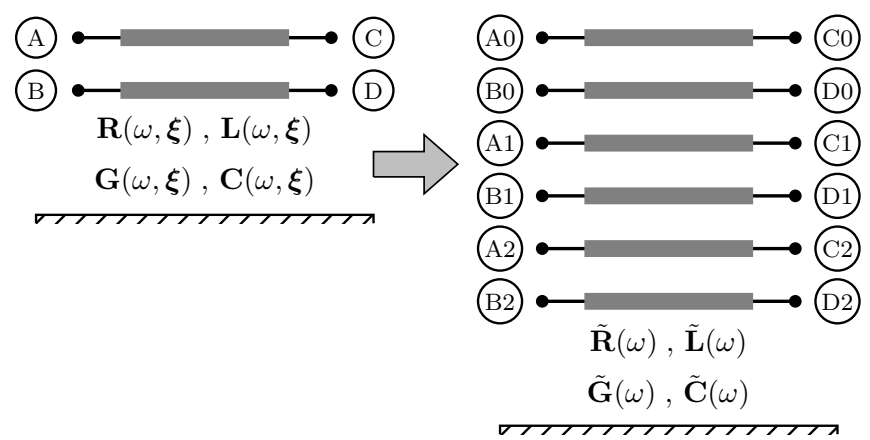

Fig. 1. Pictorial illustration of the augmentation of a MTL-element: original stochastic circuit element (left) and corresponding augmented deterministic element (right).

instance of the circuit, by a larger MTL described by the p.u.l. parameters $\tilde{\mathbf{R}}, \tilde{\mathbf{L}}, \tilde{\mathbf{G}}$ and $\tilde{\mathbf{C}}$.

Concerning the inclusion of other kinds of (lumped) circuit elements, in this paper and without loss of generality we limit ourselves to consider deterministic lumped components only. Hence, the variability is exclusively provided by the distributed elements, as this represents the main achievement of this paper. The derivation of deterministic constitutive relations for the voltage and current coefficients of stochastic lumped elements has already been addressed in [7] or, alternatively, in [11].

We consider a lumped element connecting two nodes, indicated as $C$ and $D$, and described by a Norton equivalent:

$$
I(\omega, \boldsymbol{\xi})=Y(\omega)\left[V_{C}(\omega, \boldsymbol{\xi})-V_{D}(\omega, \boldsymbol{\xi})\right]-A(\omega),
$$

where $Y$ and $A$ are the equivalent admittance and current source, respectively. It should be noted that voltages and currents are still stochastic due to the presence in the network of other random circuit components. Again, the application of the SGM allows to derive deterministic equations for the PC-coefficients, here gathered and written in matrix form:

$\tilde{\mathbf{I}}(\omega)=Y(\omega) \cdot \mathbb{I}_{P+1}\left[\tilde{\mathbf{V}}_{C}(\omega)-\tilde{\mathbf{V}}_{D}(\omega)\right]-[A(\omega), 0, \ldots, 0]^{T}$,

where $\mathbb{I}_{P+1}$ is the $(P+1) \times(P+1)$ identity matrix, and the vectors denoted with a tilde collect the PC-coefficients for the node voltages and the branch currents. Equations (11) are decoupled and define the behavior of the branches connecting the $2(P+1)$ nodes which result from the expansion of node voltages $V_{C}$ and $V_{D}$, as shown in Fig. 2 for $P=2$. The circuit interpretation consists in the replication of the equivalent admittance on the additional branches. On the other hand, no replication is required for the equivalent source.

The reasoning in this section equivalently applies in time domain and outlines a practical rule to derive the overall augmented circuit, which is obtained by direct construction and connection of the new elements consistently with the original circuit topology [11]: stochastic lines are replaced by their augmented counterparts, whilst deterministic lumped elements are replicated, as shown in Figs. 1 and 2, respectively. For the PC simulation, a single solution of this network provides the PC-coefficients for the stochastic circuit variables.
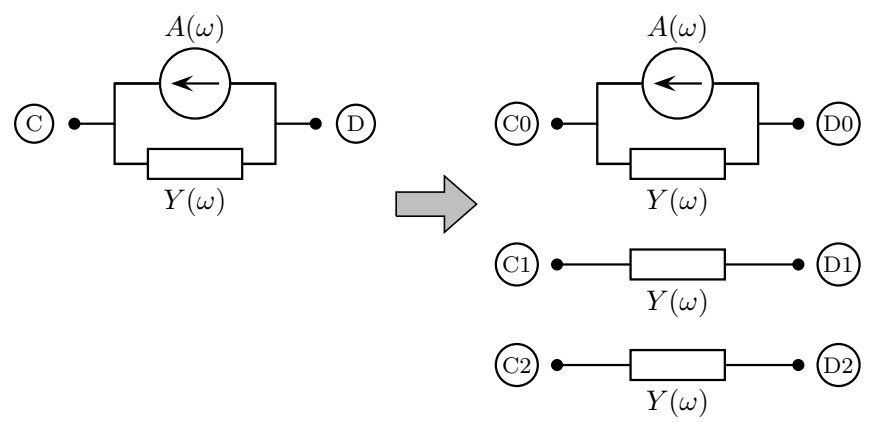

Fig. 2. Illustration of the augmentation of a non-stochastic lumped element.

\section{COMputation AND SPICE-TyPe Description OF The Augmented TRAnsmission-Line Models}

The PC approach requires the computation of the expansion coefficients $\mathbf{R}_{k}, \mathbf{L}_{k}, \mathbf{G}_{k}$, and $\mathbf{C}_{k}$ to be used in (9) for the construction of the augmented p.u.l. matrices $\tilde{\mathbf{R}}, \tilde{\mathbf{L}}, \tilde{\mathbf{G}}$ and $\tilde{\mathbf{C}}$. Such expansion coefficients can be obtained according to the classical projection theorem by using the inner product (2), i.e.

$$
\mathbf{A}_{k}(\omega)=\left\langle\mathbf{A}, \phi_{k}\right\rangle=\int_{\Omega} \mathbf{A}(\omega, \boldsymbol{\xi}) \phi_{k}(\boldsymbol{\xi}) w(\boldsymbol{\xi}) d \boldsymbol{\xi},
$$

where $\mathbf{A}(\omega, \boldsymbol{\xi})$ is the outcome of some function or tool for the computation of the p.u.l. parameters. Efficient evaluation of (12) can be achieved by means of Gaussian quadratures [15], i.e. as a weighted sum of discrete values computed at pre-defined points:

$$
\int_{\Omega} \mathbf{A}(\omega, \boldsymbol{\xi}) \phi_{k}(\boldsymbol{\xi}) w(\boldsymbol{\xi}) d \boldsymbol{\xi} \approx \sum_{k=0}^{K} \mathbf{A}\left(\omega, \boldsymbol{\xi}_{k}\right) \phi_{k}\left(\boldsymbol{\xi}_{k}\right) w_{k},
$$

where the weights $w_{k}$ are related to the weighting function $w(\boldsymbol{\xi})$ and $K+1=(p+1)^{r}$. The points $\boldsymbol{\xi}_{k}$ are a tensor product combination of the zeros of the adopted class of polynomials.

As far as the practical implementation is concerned, advanced SPICE-type circuit analysis tools, such as HSPICE or Agilent's ADS, include the "W-element" for the modeling of lossy and dispersive MTLs [16]. The W-element is a circuit component which provides a numerical solution of the telegrapher's equations (6). It generally accepts as inputs static $\mathbf{R}, \mathbf{L}, \mathbf{G}$ and $\mathbf{C}$ matrices or, for dispersive lines, tabulated frequency data. However, due to the inherent physical symmetry of the RLGC-matrices, the W-element only requires the user to specify the lower triangular part of these matrices. In Section III, we have shown how the PC-method leads to an augmented transmission-line model, described by an appropriately extended set of RLGC-matrices. In order to be able to implement it into HSPICE via the W-element, we must assure that these extended matrices are also symmetric. This is not trivial and in order to be accomplished, as we have already pointed out in [12], the polynomials $\left\{\phi_{k}\right\}$ in (2) should not be merely orthogonal but orthonormal. This is a crucial step for the SPICE-implementation put forward in this paper.

It is also worth mentioning that HSPICE provides an internal field solver (FS), which is capable of computing lossy and dispersive p.u.l. parameters and simulating a transmission line 
upon the description of its cross-section [16]. The FS can be combined with the available feature for Monte Carlo simulations, thus allowing to perform a statistical analysis of circuits containing transmission lines with stochastic cross-sectional parameters entirely in the HSPICE environment. Although it is in general not accurate for structures characterized by complex wave phenomena, such as on-chip lines, the use of the FS and the consequent creation of consistent PC-based transmissionline models can be useful in many practical situations.

The FS basically computes six frequency independent p.u.l. matrices: the DC inductance $\mathbf{L}_{0}[\mathrm{H} / \mathrm{m}]$, capacitance $\mathbf{C}_{0}[\mathrm{~F} / \mathrm{m}]$, resistance $\mathbf{R}_{0}[\Omega / \mathrm{m}]$, and conductance $\mathbf{G}_{0}[\mathrm{~S} / \mathrm{m}]$ matrices, as well as the skin effect resistance matrix $\mathbf{R}_{s}[\Omega /(\mathrm{m} \sqrt{\mathrm{Hz}})]$ and the dielectric loss conductance matrix $\mathbf{G}_{d}[\mathrm{~S} /(\mathrm{m} \cdot \mathrm{Hz})]$. These six matrices are combined in a frequency-dependent way to obtain the dispersive behavior. Also, they are obtainable as an output or supplied as an input to a W-element (the "RLGC model"). For the creation of the augmented transmission-line model, it is alternatively possible to project $\mathbf{L}_{0}, \mathbf{C}_{0}, \mathbf{R}_{0}, \mathbf{G}_{0}$, $\mathbf{R}_{s}$ and $\mathbf{G}_{d}$ according to (13) and compute their augmented counterparts $\tilde{\mathbf{L}}_{0}, \tilde{\mathbf{C}}_{0}, \tilde{\mathbf{R}}_{0}, \tilde{\mathbf{G}}_{0}, \tilde{\mathbf{R}}_{s}$ and $\tilde{\mathbf{G}}_{d}$ with (9). These are then supplied in place of the tabular model to create a PC-based model which is consistent with the internal FS and therefore with the HSPICE Monte Carlo analysis.

\section{VALidation AND Numerical Results}

In this section, the proposed approach is applied to the simulation of several lossy and dispersive interconnect structures. As time-domain analysis of such structures is usually more challenging, only transient simulations are considered and carried out using HSPICE. All the simulations are performed on an ASUS U30S laptop with an Intel(R) Core(TM) i3$2330 \mathrm{M}$, CPU running at $2.20 \mathrm{GHz}$ and $4 \mathrm{~GB}$ of RAM.

\section{A. Inverted Embedded Microstrip Line}

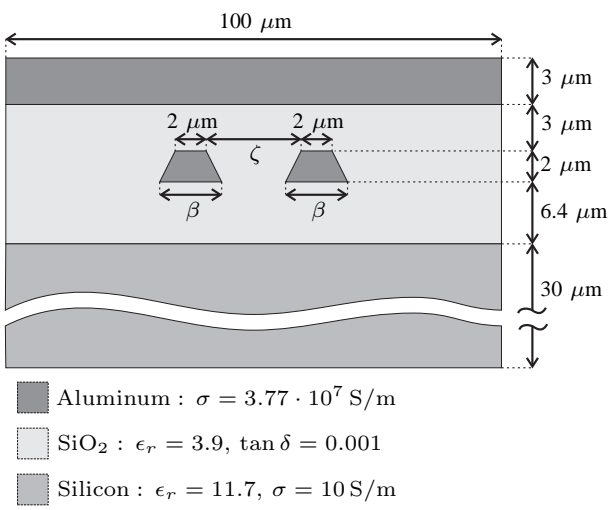

Fig. 3. Cross-section of the coupled on-chip IEM line (not to scale).

The first example we consider is that of the coupled inverted embedded microstrip (IEM) on-chip line shown in Fig. 3. Due to its tiny dimensions and presence of semiconductors, such a configuration is characterized by high losses and a complex frequency behavior. Therefore, its p.u.l. parameters cannot be accurately computed by means of the HSPICE FS. A macromodel for the p.u.l. impedance and admittance matrices of this coupled line is available [10], based on accurate field simulations and parameterized with respect to the base width $\beta$ and the separation of the conductors $\zeta$. Variations in the base width account for the trapezoidal profile resulting from the etching process, while variations in the separation describe the variability of the photolitographic process. Because of the randomness of these manufacturing steps, it is reasonable to model such parameters as two independent Gaussian random variables. Hence, the optimal choice for the basis $\left\{\phi_{k}\right\}$ is represented in this case by a properly-rescaled version of Hermite polynomials [12]. Here, a mean value of $2 \mu \mathrm{m}$ and a relative standard deviation of $10 \%$ are considered for $\beta$, whereas $\zeta$ has mean $5 \mu \mathrm{m}$ and a standard deviation of $5 \%$.

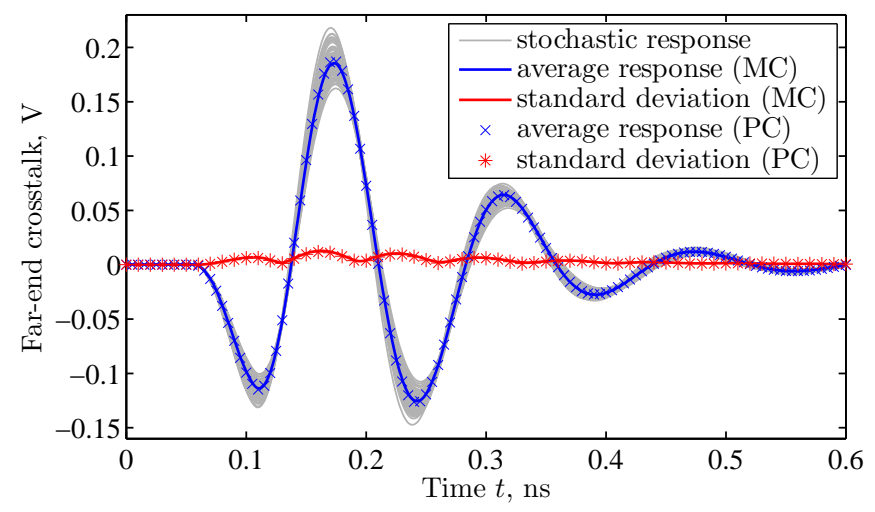

Fig. 4. Far-end crosstalk voltage on the coupled IEM line of Fig. 3. Gray lines: subset of 100 samples from Monte Carlo simulation; other solid lines: mean value (blue) and standard deviation (red) estimated with Monte Carlo analysis. Markers $(x)$ and $(*)$ indicate the same statistical information obtained from the PC-expansions.

The analyticity of the parametric macromodel allows to quickly compute a tabular PC-augmented model for the p.u.l. parameters of the IEM line [10], and this can be supplied as an input to a W-element in a HSPICE netlist. Also, the macromodel is randomly sampled to perform repeated Monte Carlo simulations. For each run, a different tabular input is generated and a new HSPICE simulation is launched. It is important to note that the macromodel also allows to speedup the Monte Carlo simulation itself, as the computation of an actual sample of the p.u.l. parameters by means of the accurate external solver would be much slower.

Fig. 4 shows the stochastic transient simulation of the farend crosstalk for the case in which one line is excited by a $1-\mathrm{V}$ step source with a 50-ps risetime and internal impedance of $1 \Omega$, and terminated by a capacitance of $1 \mathrm{pF}$. The other line is quiet and is also terminated by a $1-\Omega$ resistance and a 1 -pF capacitance at the near- and far-end sides, respectively. The gray lines show the spread of the response resulting from a subset of 100 Monte Carlo simulations. The blue line is the average response, while the red line is the standard deviation, both estimated from 1000 Monte Carlo samples. The markers depict the same statistical information obtained from a secondorder PC simulation, showing excellent agreement. The local maxima of the standard deviation indicate points where the 
fluctuation of the response is larger.

As far as the computational times are concerned, the Monte Carlo analysis required 29 mins, whereas the PC circuit simulation took $2.7 \mathrm{~s}$. We can thus conclude that, for this first example, the achieved speed-up is $640 \times$, without any compromise on the accuracy. It is relevant to point out that the created model is not restricted to this specific simulation, but can be stored and re-used for other circuits involving the same stochastic line structure. Therefore, the time required to generate the augmented tabular model, which amounts to $53 \mathrm{~s}$, is not included in the above figures.

\section{B. Transmission-Line Network with Single Lines}

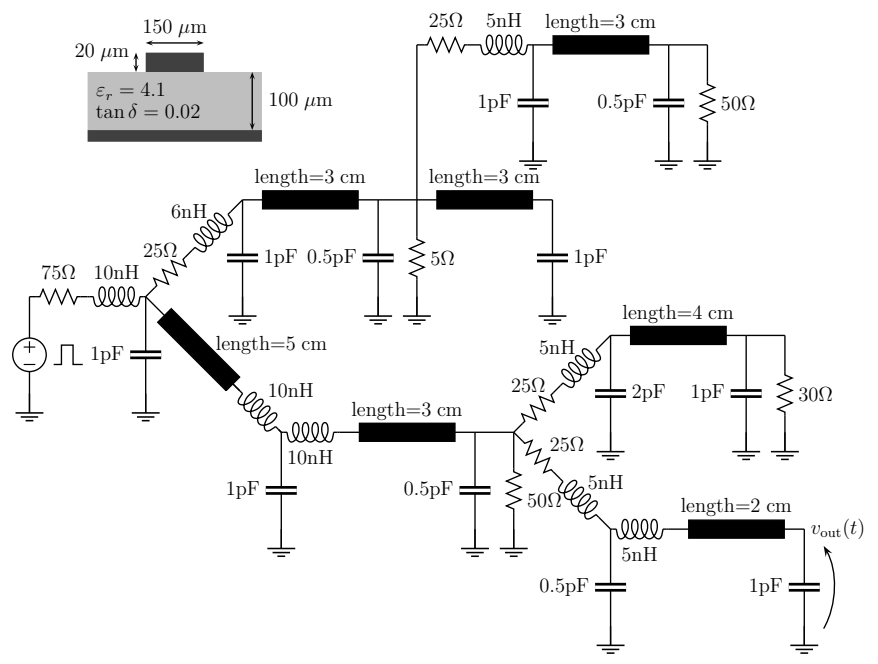

Fig. 5. Transmission-line network with single microstrip traces and corresponding line cross-section.

The second example deals with the interconnect network illustrated in Fig. 5, whose topology is inspired by [18]. In this case, the voltage source is a $1-\mathrm{V}$ pulse with rise/fall times of $200 \mathrm{ps}$ and width of $2.6 \mathrm{~ns}$. The displayed microstrip cross-section, with copper traces, is considered for the transmission-line segments. The variability is here provided by the thickness, permittivity and loss tangent of the board substrate, which is shared by all the lines. The HSPICE FS is assumed to be accurate enough for the characterization of this transmission line geometry, which is then implemented as a W-element characterized by the description of its crosssection.

A Monte Carlo simulation with 1000 samples is run using the available HSPICE feature and by considering a $10 \%$ variation of the above parameters with respect to their nominal values. The plot in Fig. 6 shows the stochastic transient response of the voltage $v_{\text {out }}(t)$ transmitted to the far-end side of the network, together with its average value and standard deviation. These statistical parameters are also obtained from a PC simulation, which again reveals perfect agreement. In this case, a second-order PC-based RLGC model described by augmented $\tilde{\mathbf{L}}_{0}, \tilde{\mathbf{C}}_{0}, \tilde{\mathbf{R}}_{0}, \tilde{\mathbf{R}}_{s}, \tilde{\mathbf{G}}_{0}$ and $\tilde{\mathbf{G}}_{d}$ matrices is computed by means of a Gauss-Hermite quadrature and 27 calls to the FS. This step took $22.2 \mathrm{~s}$. The circuit simulation

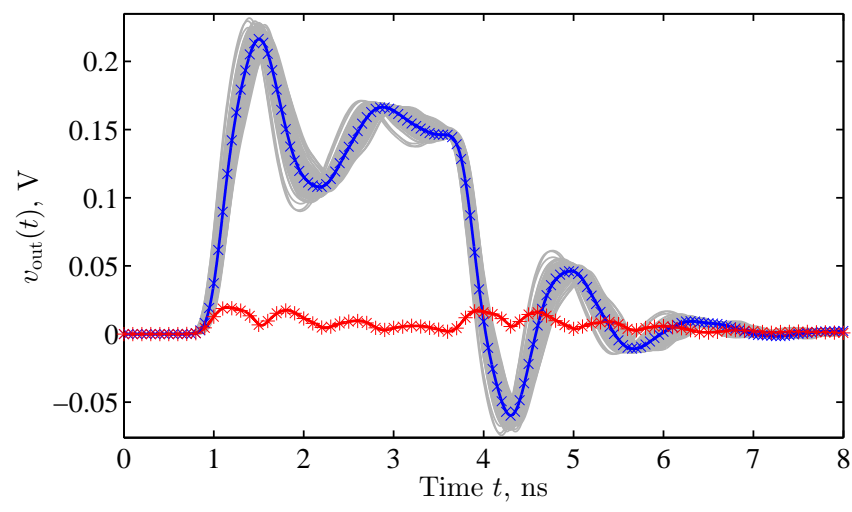

Fig. 6. Voltage $v_{\text {out }}(t)$ transmitted to the far end of the transmission-line network of Fig. 5. Curves identification as in the inset of Fig. 4

of the augmented network required $7.5 \mathrm{~s}$ instead, whereas the Monte Carlo simulation took 38 mins 25 s. For this second example, the speed-up in circuit simulation is $310 \times$.

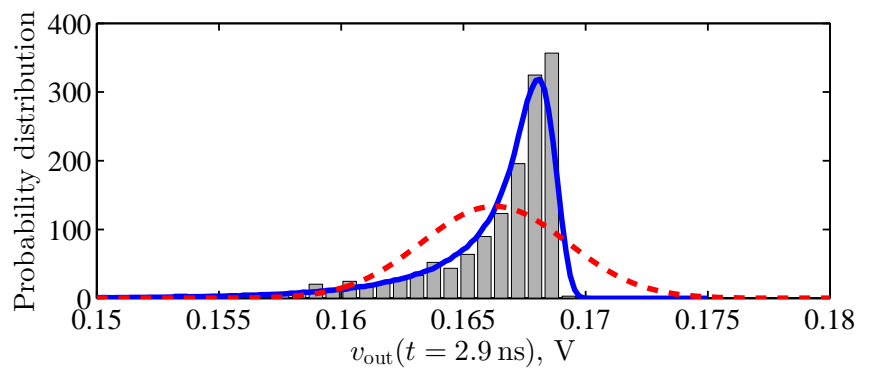

Fig. 7. Probability density function of $v_{\text {out }}(t)$ computed at $t=2.9 \mathrm{~ns}$ from the Monte Carlo samples (bars) and polynomial chaos expansions (blue line). Dashed red line: corresponding Gaussian distribution.

It is worth noting that, despite the normal distribution of the random input parameters, the distribution of the response is in general not Gaussian and therefore average value and standard deviation are not sufficient for a complete statistical characterization. For instance, Fig. 7 displays the PDF at $t=2.9 \mathrm{~ns}$, computed from the Monte Carlo samples (bars) and from the PC-expansion (blue line). For comparison, the dashed red line is the Gaussian distribution corresponding to the estimated mean value and standard deviation. It should be noted that the small number of Monte Carlo samples (the number of samples is typically of the order of 10000) leads to a poor resolution of the PDF compared to the result obtained with the PC solution.

\section{Transmission-Line Network with Coupled Lines}

We now consider the transmission-line network displayed in Fig. 8 [18]. The coupled lines again have a microstrip crosssection with random geometrical parameters, i.e. the copper trace width, trace-to-trace separation, trace thickness, and substrate thickness have $10 \%$ independent relative standard deviations. Furthermore, the substrate has a permittivity of 3.7 and a loss tangent of 0.02 . The FS is used to characterize the electrical behavior of the transmission-line sections. The 


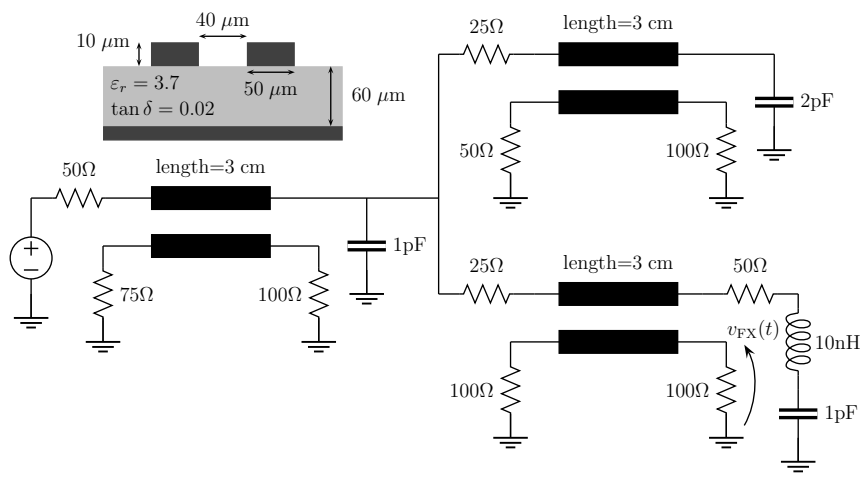

Fig. 8. Transmission-line network with coupled microstrip traces and corresponding line cross-section.

voltage source is a Gaussian pulse of peak amplitude of $1 \mathrm{~V}$ and a width of about $0.177 \mathrm{~ns}$ at half amplitude.

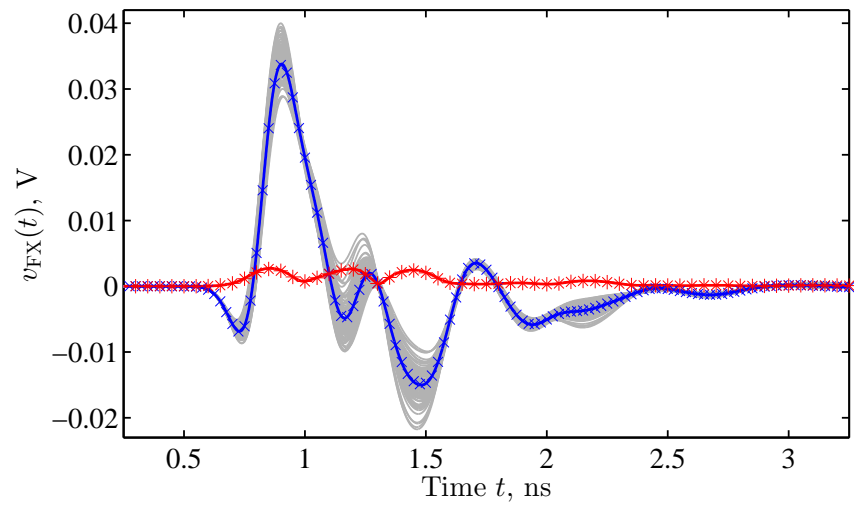

Fig. 9. Far-end crosstalk voltage $v_{\mathrm{FX}}(t)$ in the transmission-line network of Fig. 8. Curves identification as in the inset of Fig. 4.

Fig. 9 shows the results obtained with the HSPICE Monte Carlo analysis (1000 samples) of the far-end crosstalk voltage $v_{\mathrm{FX}}(t)$, which required 14 mins $15 \mathrm{~s}$. The comparison with a PC simulation, taking $14.2 \mathrm{~s}$ and based on a secondorder augmented RLGC model, confirms the accuracy of the proposed technique and shows a $60 \times$ speed-up. The generation of the augmented model required 55 s. Finally, Fig. 10 displays the PDF for the crosstalk peak, occurring at $t=0.9 \mathrm{~ns}$. Again, it is possible to appreciate the better reproduction of the distribution provided by the PC result.

\section{CONCLUSions}

This paper proposes an alternative approach to Monte Carlo circuit simulation of high-speed interconnects affected by random parameter variability. The method is based on the representation of stochastic circuit variables in terms of orthonormalized polynomial functions and applies to lossy and dispersive multiconductor transmission lines with multiple random variables. The determination of the expansion coefficients allows a convenient extraction of statistical information and is carried out via a single simulation of a modified (augmented) circuit, which can be readily derived from the original network topology.

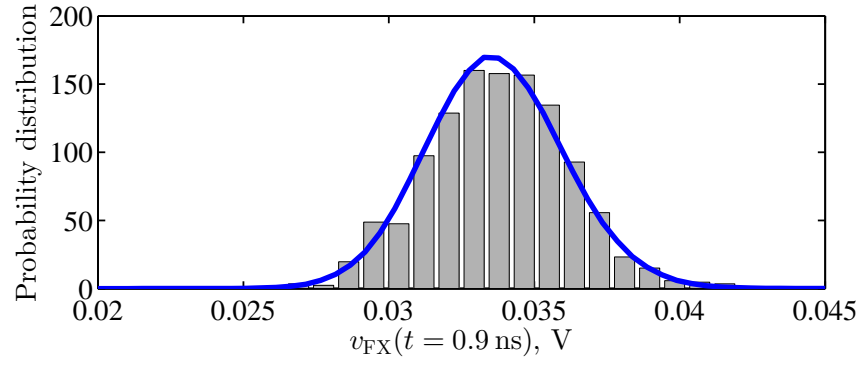

Fig. 10. Probability density function of $v_{\mathrm{FX}}(t)$ for $t=0.9$ ns. Gray bars: result of Monte Carlo analysis. Blue line: estimation from polynomial chaos expansion

The novelty of the advocated technique lies in the extension of literature stochastic circuit modeling strategies with the inclusion of lossy and dispersive distributed elements. Moreover, it takes advantage of a SPICE-type implementation to perform transient simulations and facilitate the analysis of arbitrary network topologies without the need for customized software. This kind of analysis was not possible with previouslypresented PC-based techniques.

The approach is validated by means of three application examples involving on-chip and on-board lines. Although the efficiency with respect to Monte Carlo depends on the number of samples considered, the PC approach turns out to be about two orders of magnitude faster even when a very small number of 1000 simulations is considered, nevertheless showing excellent accuracy on the prediction of statistical information. It is important to remark that the proposed technique can be applied to multiconductor lines having an arbitrary number of conductors, the efficiency of the approach being mainly determined by the number of terms considered for the PCexpansions.

\section{REFERENCES}

[1] T. Mikazuki and N. Matsui, "Statistical design techniques for high-speed circuit boards with correlated structure distributions," IEEE Trans. Compon. Packag. Manuf. Technol. A, vol. 17, no. 1, pp. 159-165, Mar. 1994.

[2] A. H. Zaabab, Qi-Jun Zhang, and M. Nakhla, "A neural network modeling approach to circuit optimization and statistical design," IEEE Trans. Microw. Theory Tech., vol. 43, no. 6, pp. 1349-1358, Jun. 1995.

[3] R. G. Ghanem and P. D. Spanos, Stochastic Finite Elements. A Spectral Approach. New York: Springer-Verlag, 1991.

[4] D. Xiu and G. E. Karniadakis, "The Wiener-Askey polynomial chaos for stochastic differential equations," SIAM J. Sci. Comput., vol. 24, no. 2, pp. 619-622, 2002.

[5] S. Vrudhula, J. M. Wang, and P. Ghanta, "Hermite polynomial based interconnect analysis in the presence of process variations," IEEE Trans. Comput.-Aided Des. Integr. Circuits Syst., vol. 25, no. 10, pp. 2001-2011, Oct. 2006.

[6] N. Mi, S. X.-D. Tan, Y. Cai, and X. Hong, "Fast variational analysis of on-chip power grids by stochastic extended Krylov subspace method," IEEE Trans. Comput.-Aided Des. Integr. Circuits Syst., vol. 27, no. 11, pp. 1996-2006, Nov. 2008.

[7] K. Strunz and Q. Su, "Stochastic formulation of SPICE-type electronic circuit simulation using polynomial chaos," ACM Trans. Model. Comput. Sim. vol. 18, no. 4, pp. 15:1-15:23, Sep. 2008.

[8] X. Li, J. M. Wang, W. Tang, and H. Wu, "Stochastic analysis for crosstalk noise of coupled interconnects with process variations," in Proc. IEEE Int. Conf. Integr. Circ. Des. Tech. Tut., Austin, TX, Jun. 2008, pp. 289292. 
[9] I. S. Stievano, P. Manfredi, and F. G. Canavero, "Parameters variability effects on multiconductor interconnects via Hermite polynomial chaos," IEEE Trans. Compon. Packag. Technol., vol. 1, no. 8, pp. 1234-1239, Aug. 2011.

[10] D. Vande Ginste, D. De Zutter, D. Deschrijver, T. Dhaene, P. Manfredi, and F. Canavero, "Stochastic modeling-based variability analysis of onchip interconnects," IEEE Trans. Compon. Packag. Technol., vol. 2, no. 7, pp. 1182-1192, Jul. 2012.

[11] P. Manfredi, I. S. Stievano, and F. G. Canavero, "Alternative SPICE implementation of circuit uncertainties based on orthogonal polynomials," in Proc. IEEE 20th Conf. Elect. Perform. Electron. Packag. Syst., San Jose, CA, Oct. 2011, pp. 41-44.

[12] P. Manfredi, D. Vande Ginste, D. De Zutter, and F. G. Canavero, "Improved Polynomial Chaos Discretization Schemes to Integrate Interconnects into Design Environments," accepted for publication in IEEE Microw. Wireless Compon. Lett.

[13] P. Manfredi, D. Vande Ginste, D. De Zutter, and F. G. Canavero, "Frequency- and time-domain stochastic analysis of lossy and dispersive interconnects in a SPICE-like environment," in Proc. IEEE 21th Conf. Elect. Perform. Electron. Packag. Syst., Tempe, AZ, Oct. 2012, pp. 65-68.

[14] M. Loève, Probability Theory. 4th edition, New York: Springer-Verlag, 1977.

[15] W. H. Press, S. A. Teukolsky, W. T. Vetterling, and B. P. Flannery, "Numerical Recipes: The Art of Scientific Computing". 3rd edition, New York: Cambridge University Press, 2007.

[16] "HSPICE®User Guide: Signal Integrity”. Version B-2008.09, September 2008

[17] M. Abramowitz and I. A. Stegun, Handbook of Mathematical Functions. New York: Dover, 1972.

[18] T. K. Tang and M. S. Nakhla, "Analysis of high-speed VLSI interconnects using the asymptotic waveform evaluation technique," IEEE Trans. Comput.-Aided Des., vol. 11, no. 3, pp. 341-352, Mar. 1992. 